\title{
Coefficient Inequalities of Analytic Functions Related to Robertson Functions
}

\author{
Muhammad Arif and Mumtaz Ali \\ Department of Mathematics, Abdul Wali Khan University, 23200 Mardan, Khyber Pakhtunkhwa, Pakistan \\ Correspondence should be addressed to Muhammad Arif; marifmaths@yahoo.com \\ Received 5 January 2013; Revised 19 March 2013; Accepted 2 April 2013 \\ Academic Editor: S. T. Ali
}

Copyright (C) 2013 M. Arif and M. Ali. This is an open access article distributed under the Creative Commons Attribution License, which permits unrestricted use, distribution, and reproduction in any medium, provided the original work is properly cited.

We introduce and study a subclass of analytic functions related to Robertson functions. Here we discuss the coefficient estimate for function in this class.

\section{Introduction}

Let $A$ be the class of functions $f$ of the form

$$
f(z)=z+\sum_{n=2}^{\infty} a_{n} z^{n}
$$

which are analytic in the open unit disc $E=\{z:|z|<1\}$. Also let $S^{*}$ and $C$ denote the well-known classes of starlike and convex functions, respectively.

For any two analytic functions $f$ given by (1) and $g$ with

$$
g(z)=z+\sum_{n=2}^{\infty} b_{n} z^{n}, \quad \text { for } z \in E,
$$

the convolution (Hadamard product) is given by

$$
(f \star g)(z)=z+\sum_{n=2}^{\infty} a_{n} b_{n} z^{n}, \quad \text { for } z \in E .
$$

Using the concept of convolution, Ruscheweyh [1] introduced a differential operator $D^{\delta}$ given by

$$
\begin{array}{r}
D^{\delta} f(z)=\frac{z}{(1-z)^{\delta+1}} * f(z)=z+\sum_{n=2}^{\infty} \varphi_{n}(\delta) a_{n} z^{n}, \\
(\delta>-1)
\end{array}
$$

with

$$
\varphi_{n}(\delta)=\frac{(\delta+1)_{n-1}}{(n-1) !},
$$

where $(x)_{n}$ is a Pochhammer symbol given as

$$
(x)_{n}= \begin{cases}1, & n=0, \\ x(x+1)(x+2) \cdots(x+n-1), & n \in \mathbb{N} .\end{cases}
$$

It is obvious that $D^{0} f(z)=f(z), D^{1} f(z)=z f^{\prime}(z)$, and

$$
D^{n} f(z)=\frac{z\left(z^{n-1} f(z)\right)^{(n)}}{n !}, \quad \forall \delta=n \in N_{0}=\{0,1,2, \ldots\} .
$$

The following identity can easily be established:

$$
(\delta+1) D^{\delta+1} f(z)=\delta D^{\delta} f(z)+z\left(D^{\delta} f(z)\right)^{\prime} .
$$

Now with the help of Ruscheweyh derivative, we define a class $V D_{\lambda}(\alpha, \beta, b, \delta)$ of analytic functions as follows.

Definition 1. Let $f(z) \in A$. Then, $f(z) \in V D_{\lambda}(\alpha, \beta, b, \delta)$, if and only if

$$
\begin{aligned}
\operatorname{Re}\left\{e^{i \lambda}\left(1-\frac{2}{b}+\frac{2}{b} \frac{D^{\delta+1} f(z)}{D^{\delta} f(z)}\right)\right\} \\
>\alpha\left|\frac{2}{b}\left(\frac{D^{\delta+1} f(z)}{D^{\delta} f(z)}-1\right)\right|+\beta \cos \lambda,
\end{aligned}
$$

where $\alpha \geq 0,0 \leq \beta<1, \delta>-1, \lambda$ is real with $|\lambda|<\pi / 2$, and $b \in \mathbb{C} \backslash\{0\}$. 
By giving specific values to $\alpha, \beta, \lambda, b$, and $\delta$ in $V D_{\lambda}(\alpha, \beta, b, \delta)$, we obtain many important subclasses studied by various authors in earlier papers, see for details [2-5], and list some of them as follows:

(i) $V D_{\lambda}(0,0,2,0) \equiv S_{\lambda}^{*}$ and $V D_{\lambda}(0,0,1,1) \equiv K_{\lambda}$, studied by Spacek [6] and Robertson [7], respectively; for the advancement work, see $[8,9]$.

(ii) $V D_{0}(\alpha, \beta, 2,0) \equiv S D(\alpha, \beta)$ and $V D_{0}(\alpha, \beta, 1,1) \equiv$ $K D(\alpha, \beta)$, studied by both Owa et al. and Shams et al. $[10,11]$.

(iii) $V D_{\lambda}(1,0,2,0) \equiv \operatorname{USP}(\lambda), V D_{\lambda}(1,0,1,1) \equiv \operatorname{UCSP}(\lambda)$, introduced by Ravichandran et al. [12].

(iv) $V D_{0}(\alpha, \beta, b, \delta) \equiv V D(\alpha, \beta, b, \delta)$, considered by Latha [13].

(v) $V D_{0}(0, \beta, 2,0) \equiv S^{*}(\beta), V D_{0}(0, \beta, 1,1) \equiv C(\beta)$, the well-known classes of starlike and convex functions of order $\beta$.

From the above special cases, we note that this class provides a continuous passage from the class of starlike functions to the class of convex functions.

We will assume throughout our discussion, unless otherwise stated, that $\alpha \geq 0,0 \leq \beta<1, \delta>-1, \lambda$ is real with $|\lambda|<\pi / 2$, and $b \in \mathbb{C} \backslash\{0\}$.

\section{Some Properties of the Class $V D_{\lambda}(\alpha, \beta, b, \delta)$}

Theorem 2. If $f(z) \in V D_{\lambda}(\alpha, \beta, b, \delta)$ with $0 \leq \alpha \leq \beta$, then

$$
f(z) \in V D_{\lambda}\left(0,\left(\frac{\beta-\alpha}{1-\alpha}\right), b, \delta\right) .
$$

Proof. Since $\operatorname{Re} w \leq|w|$ for any complex number $w, f(z) \in$ $V D_{\lambda}(\alpha, \beta, b, \delta)$ implies that

$$
\begin{aligned}
\operatorname{Re} & \left\{e^{i \lambda}\left(1-\frac{2}{b}+\frac{2}{b} \frac{D^{\delta+1} f(z)}{D^{\delta} f(z)}\right)\right\} \\
> & \alpha\left|\frac{2}{b} \frac{D^{\delta+1} f(z)}{D^{\delta} f(z)}-\frac{2}{b}\right|+\beta \cos \lambda \\
\geq & \alpha \operatorname{Re}\left\{e^{i \lambda}\left(1-\frac{2}{b}+\frac{2}{b} \frac{D^{\delta+1} f(z)}{D^{\delta} f(z)}\right)\right\} \\
+ & (\beta-\alpha) \cos \lambda
\end{aligned}
$$

which implies that

$$
\begin{array}{r}
\operatorname{Re}\left\{e^{i \lambda}\left(1-\frac{2}{b}+\frac{2}{b} \frac{D^{\delta+1} f(z)}{D^{\delta} f(z)}\right)\right\} \\
>\frac{(\beta-\alpha) \cos \lambda}{(1-\alpha)}, \quad(z \in E) .
\end{array}
$$

And hence, we obtain the required result.

Put $\lambda=0, b=2$, and $\delta=0$ in Theorem 2; we obtain the following result.
Corollary 3 (see [10]). If $f(z) \in S D(\alpha, \beta)$ with $0 \leq \alpha \leq \beta$, then

$$
f(z) \in S^{*}\left(\frac{\beta-\alpha}{1-\alpha}\right) .
$$

Set $\lambda=0, b=1$, and $\delta=1$ in Theorem 2; one has the following result.

Corollary 4 (see [10]). If $f(z) \in K D(\alpha, \beta)$ with $0 \leq \alpha \leq \beta$, then

$$
f(z) \in K\left(\frac{\beta-\alpha}{1-\alpha}\right) .
$$

Theorem 5. If $f(z) \in V D_{\lambda}(\alpha, \beta, b, \delta)$, then

$$
\begin{array}{r}
\left|a_{2}\right| \leq \frac{|b||\eta|}{|1-\alpha|}, \\
\left|a_{n}\right| \leq \frac{(\delta+1)|b||\eta|}{(n-1)|1-\alpha| \varphi_{n}(\delta)} \prod_{j=1}^{n-2}\left(1+\frac{(\delta+1)|b||\eta|}{j|1-\alpha|}\right), \\
n \geq 3,
\end{array}
$$

where

$$
|\eta|=\sqrt{(1-\beta)^{2} \cos ^{2} \lambda+(1-\alpha)^{2} \sin ^{2} \lambda .}
$$

Proof. We note that for $f(z) \in V D_{\lambda}(\alpha, \beta, b, \delta)$,

$$
\operatorname{Re}\left\{e^{i \lambda}\left(1-\frac{2}{b}+\frac{2}{b} \frac{D^{\delta+1} f(z)}{D^{\delta} f(z)}\right)\right\}>\frac{(\beta-\alpha)}{(1-\alpha)} \cos \lambda,
$$

$$
z \in E
$$

Let us define the function $p(z)$ by

$$
\begin{aligned}
p(z)= & ((1-\alpha) \\
& \times\left[e^{i \lambda}\left(1-2 / b+(2 / b)\left(D^{\delta+1} f(z) / D^{\delta} f(z)\right)\right)\right] \\
& -(\beta-\alpha) \cos \lambda) \times((1-\beta) \cos \lambda+i(1-\alpha) \sin \lambda)^{-1} .
\end{aligned}
$$

Then, $p(z)$ is analytic in $E$ with $p(0)=1$ and $\operatorname{Re} p(z)>0$. Let

$$
p(z)=1+\sum_{n=1}^{\infty} p_{n} z^{n}
$$

Then, (19) can be written as

$$
\begin{aligned}
1-\frac{2}{b} & +\frac{2}{b} \frac{D^{\delta+1} f(z)}{D^{\delta} f(z)} \\
& =1+\frac{(1-\beta) \cos \lambda+i(1-\alpha) \sin \lambda}{e^{i \lambda}(1-\alpha)} \sum_{n=1}^{\infty} p_{n} z^{n},
\end{aligned}
$$

and using (8), we have

$$
\begin{gathered}
2 e^{i \lambda} \frac{(1-\alpha)}{(\delta+1)}\left(z\left(D^{\delta} f(z)\right)^{\prime}-\left(D^{\delta} f(z)\right)\right) \\
=b \eta\left(D^{\delta} f(z)\right)\left(\sum_{n=1}^{\infty} p_{n} z^{n}\right)
\end{gathered}
$$


which implies that

$$
\begin{aligned}
e^{i \lambda} a_{n}= & \frac{b \eta(\delta+1)}{2(1-\alpha)(n-1) \varphi_{n}(\delta)} \\
& \times\left\{p_{n-1}+\varphi_{2}(\delta) a_{2} p_{n-2}+\cdots+\varphi_{n-1}(\delta) a_{n-1} p_{1}\right\}
\end{aligned}
$$

where we have used (4) and (5). Now applying the coefficient estimates $\left|p_{n}\right| \leq 2$ for Caratheodory function [14], we obtain

$$
\begin{aligned}
\left|a_{n}\right| \leq & \frac{(\delta+1)|b||\eta|}{(n-1)|1-\alpha| \varphi_{n}(\delta)} \\
& \times\left[1+\varphi_{2}(\delta)\left|a_{2}\right|+\cdots+\varphi_{n-1}(\delta)\left|a_{n-1}\right|\right] .
\end{aligned}
$$

For $n=2$,

$$
\left|a_{2}\right| \leq \frac{|b||\eta|}{|1-\alpha|}
$$

which proves (15).

For $n=3$,

$$
\left|a_{3}\right| \leq \frac{(\delta+1)|b||\eta|}{2|1-\alpha| \varphi_{3}(\delta)}\left[1+\frac{(\delta+1)|b||\eta|}{|1-\alpha|}\right] .
$$

Therefore, (16) holds for $n=3$. Assume that (16) is true for all $n=3,4, \ldots k$ and consider

$$
\begin{aligned}
&\left|a_{k+1}\right| \leq \frac{(\delta+1)|b||\eta|}{(k+1-1)|1-\alpha| \varphi_{k+1}(\delta)} \\
& \times\left\{\left(1+\frac{(\delta+1)|b||\eta|}{|1-\alpha|}\right)\right. \\
&+\frac{(\delta+1)|b||\eta|}{2|1-\alpha|}\left(1+\frac{(\delta+1)|b||\eta|}{|1-\alpha|}\right) \\
&\left.+\cdots+\frac{(\delta+1)|b||\eta|}{(k-1)|| 1-\alpha \mid} \prod_{j=1}^{k-2} 1+\frac{(\delta+1)|b||\eta|}{j|1-\alpha|}\right\} \\
&=\frac{(\delta+1)|b||\eta|}{k|1-\alpha| \varphi_{k+1}(\delta)} \prod_{j=1}^{k-1}\left(1+\frac{(\delta+1)|b||\eta|}{j|1-\alpha|}\right) .
\end{aligned}
$$

Thus, the result is true for $n=k+1$, and hence by induction, (16) holds for all $n \geq 3$.

If we set $\lambda=0, b=2$, and $\delta=0$ in Theorem 5 , we get the result proved in $[10]$.

Corollary 6. If $f(z) \in S D(\alpha, \beta)$, then

$$
\begin{gathered}
\left|a_{2}\right| \leq \frac{2(1-\beta)}{|1-\alpha|} \\
\left|a_{n}\right| \leq \frac{2(1-\beta)}{(n-1)|1-\alpha|} \prod_{j=1}^{n-2}\left(1+\frac{2(1-\beta)}{j|1-\alpha|}\right), \quad(n \geq 3) .
\end{gathered}
$$

Remark 7. If we take $\alpha=0$ in Corollary 6, we have

$$
\left|a_{n}\right| \leq \frac{1}{(n-1) !} \prod_{j=2}^{n}(j-2 \beta), \quad(n \geq 2)
$$

which was proved by Robertson [15].

By setting $\lambda=0, b=1$, and $\delta=1$ in Theorem 5 , we obtain the result in [10].

Corollary 8. If $f(z) \in K D(\alpha, \beta)$, then

$$
\begin{gathered}
\left|a_{2}\right| \leq \frac{(1-\beta)}{|1-\alpha|}, \\
\left|a_{n}\right| \leq \frac{2(1-\beta)}{n(n-1)|1-\alpha|} \prod_{j=1}^{n-2}\left(1+\frac{2(1-\beta)}{j|1-\alpha|}\right), \quad(n \geq 3) .
\end{gathered}
$$

Remark 9. Letting $\alpha=0$ in Corollary 8 , we have

$$
\left|a_{n}\right| \leq \frac{1}{n !} \prod_{j=2}^{n}(j-2 \beta), \quad(n \geq 2)
$$

given by Robertson [15].

\section{Acknowledgment}

The principle author would like to thank Prof. Dr. Ihsan Ali, Vice Chancellor Abdul Wali Khan University Mardan for providing excellent research facilities and financial support.

\section{References}

[1] S. Ruscheweyh, "New criteria for univalent functions," Proceedings of the American Mathematical Society, vol. 49, no. 1, pp. 109$115,1975$.

[2] E. Aqlan, J. M. Jahangiri, and S. R. Kulkarni, "New classes of $k$ uniformly convex and starlike functions," Tamkang Journal of Mathematics, vol. 35, no. 3, pp. 261-266, 2004.

[3] S. Kanas and A. Wisniowska, "Conic regions and $k$-uniform convexity," Journal of Computational and Applied Mathematics, vol. 105, no. 1-2, pp. 327-336, 1999.

[4] S. Kanas and A. Wiśniowska, "Conic domains and starlike functions," Revue Roumaine de Mathématiques Pures et Appliquées, vol. 45, no. 4, pp. 647-657, 2000.

[5] J. Nishiwaki and S. Owa, "Certain classes of analytic functions concerned with uniformly starlike and convex functions," Applied Mathematics and Computation, vol. 187, no. 1, pp. 350$355,2007$.

[6] L. Spacek, "Prispevek k teorii funkei prostych," Časopis pro Pěstování Matematiky a Fysik, vol. 62, pp. 12-119, 1933.

[7] M. S. Robertson, "Univalent functions $f(z)$ for which $z f^{\prime}(z)$ is spirallike," The Michigan Mathematical Journal, vol. 16, pp. 97$101,1969$.

[8] M. Arif, W. Haq, and M. Ismail, "Mapping properties of generalized Robertson functions under certain integral operators," Applied Mathematics, vol. 3, no. 1, pp. 52-55, 2012. 
[9] K. I. Noor, M. Arif, and A. Muhammad, "Mapping properties of some classes of analytic functions under an integral operator," Journal of Mathematical Inequalities, vol. 4, no. 4, pp. 593-600, 2010.

[10] S. Owa, Y. Polatoğlu, and E. Yavuz, "Coefficient inequalities for classes of uniformly starlike and convex functions," Journal of Inequalities in Pure and Applied Mathematics, vol. 7, no. 5, article $160,2006$.

[11] S. Shams, S. R. Kulkarni, and J. M. Jahangiri, "Classes of uniformly starlike and convex functions," International Journal of Mathematics and Mathematical Sciences, vol. 2004, no. 55, pp. 2959-2961, 2004.

[12] V. Ravichandran, C. Selvaraj, and R. Rajagopal, "On uniformly convex spiral functions and uniformly spirallike functions," Soochow Journal of Mathematics, vol. 29, no. 4, pp. 393-405, 2003.

[13] S. Latha, "Coefficient inequalities for certain classes of Ruscheweyh type analytic functions," Journal of Inequalities in Pure and Applied Mathematics, vol. 9, no. 2, p. Article 52, 2008.

[14] C. Caratheodory, "Über den variabilitätsbereich der Fourier'schen konstanten von possitiven harmonischen funktionen," Rendiconti del Circolo Matematico di Palermo, vol. 32, pp. 193217, 1911.

[15] M. I. S. Robertson, "On the theory of univalent functions," Annals of Mathematics, vol. 37, no. 2, pp. 374-408, 1936. 


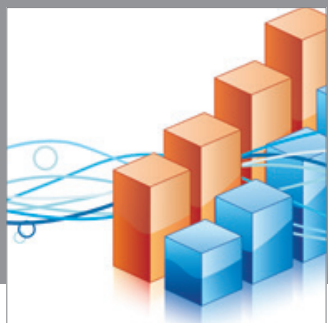

Advances in

Operations Research

mansans

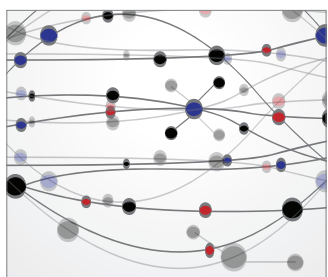

The Scientific World Journal
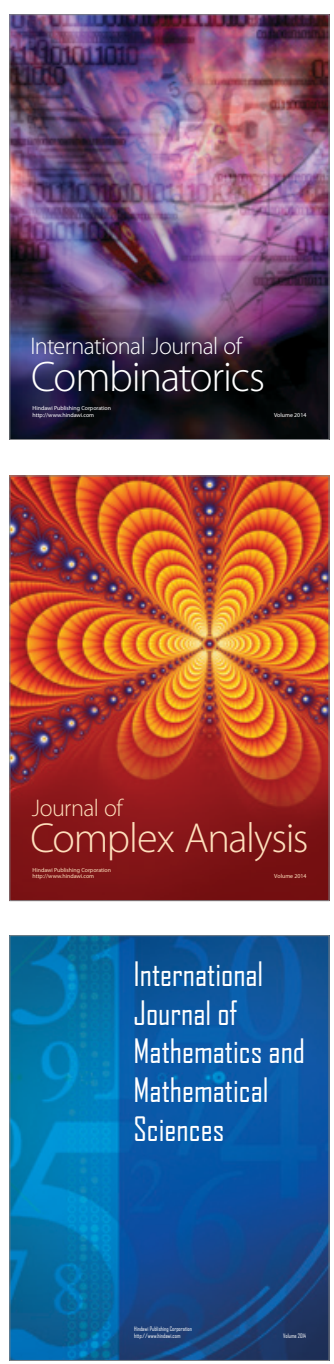
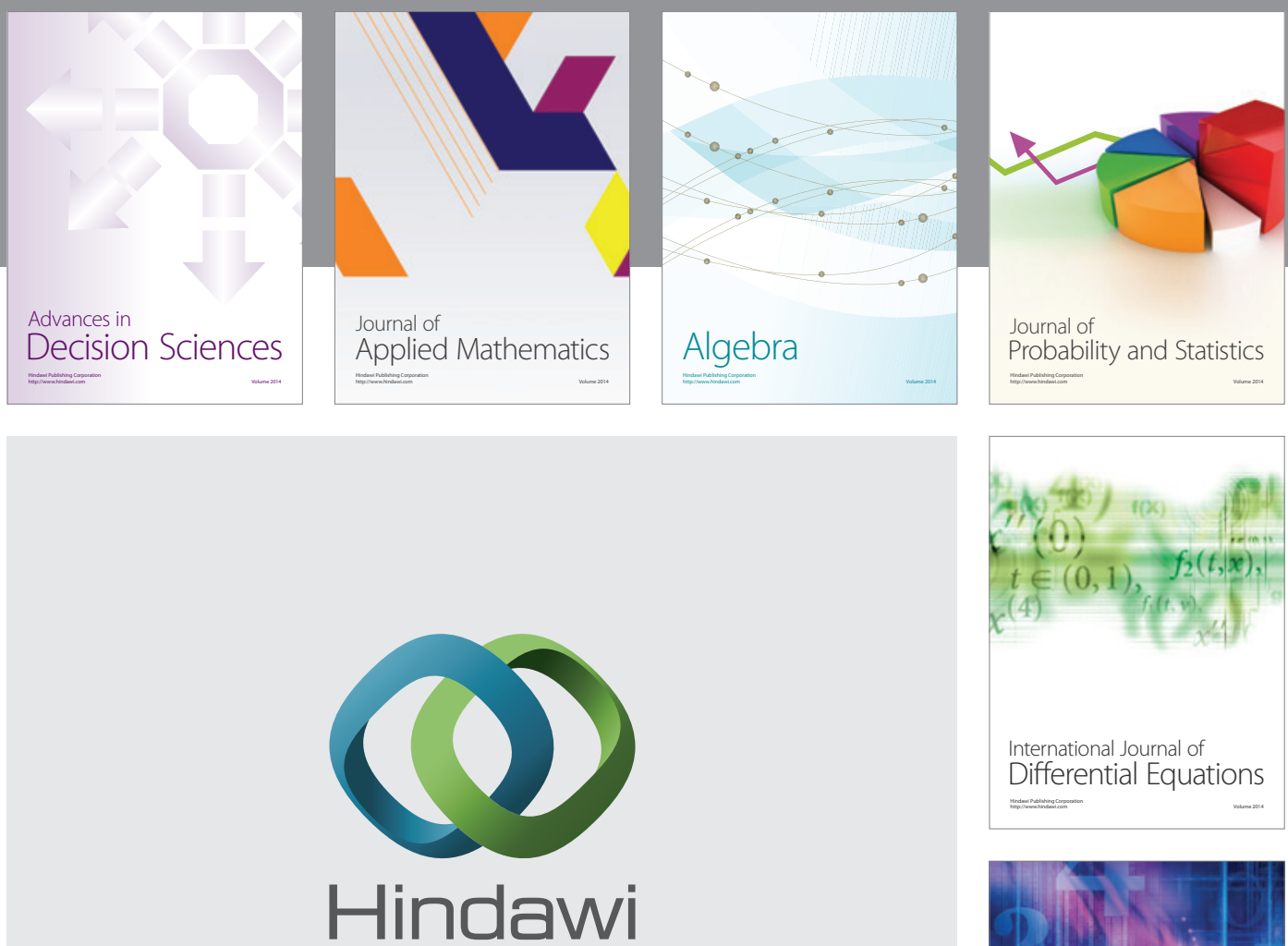

Submit your manuscripts at http://www.hindawi.com
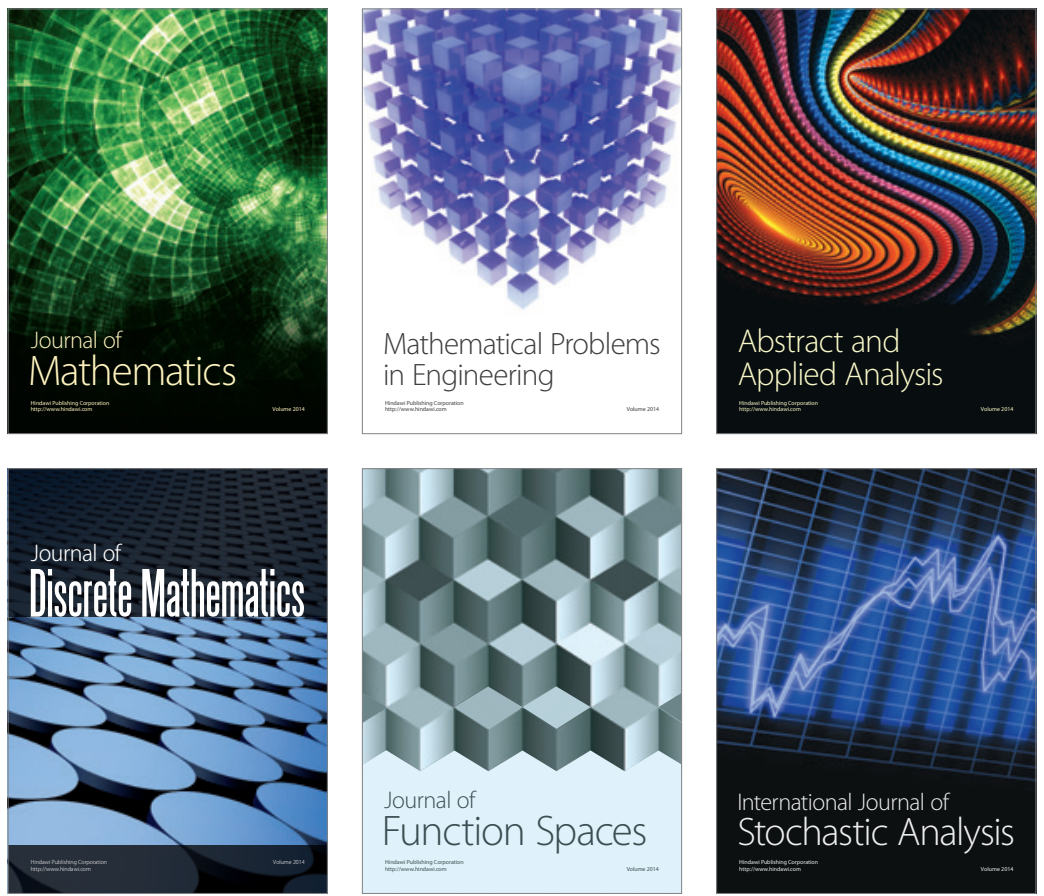

Journal of

Function Spaces

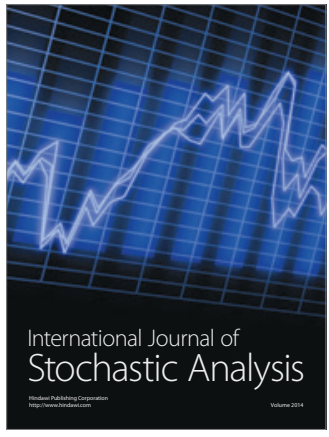

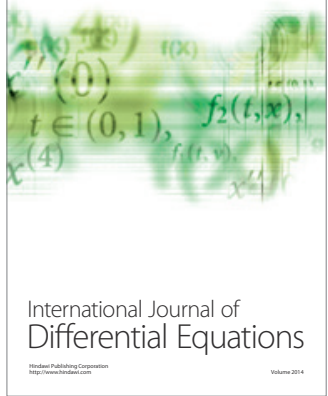
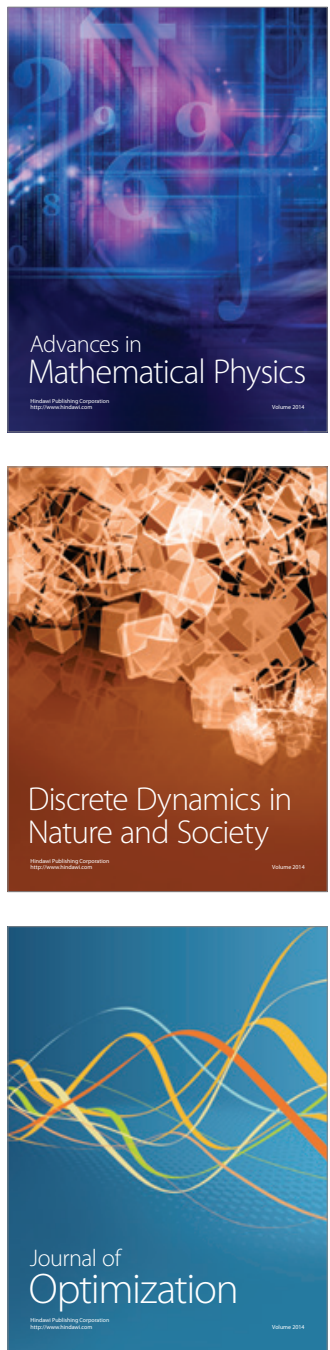